\title{
Embedding Planar Graphs at Fixed Vertex Locations
}

\author{
János Pach ${ }^{1 \star}$ and Rephael Wenger ${ }^{2 \star \star}$ \\ 1 City College, New York and the Hungarian Academy of Sciences, Budapest \\ (pach@cims.nyu.edu) \\ 2 The Ohio State University, Columbus, OH, 43210 \\ (wenger@cis.ohio-state.edu)
}

\begin{abstract}
Let $G$ be a planar graph of $n$ vertices, $v_{1}, \ldots, v_{n}$, and let $\left\{p_{1}, \ldots, p_{n}\right\}$ be a set of $n$ points in the plane. We present an algorithm for constructing in $O\left(n^{2}\right)$ time a planar embedding of $G$, where vertex $v_{i}$ is represented by point $p_{i}$ and each edge is represented by a polygonal curve with $O(n)$ bends (internal vertices.) This bound is asymptotically optimal in the worst case. In fact, if $G$ is a planar graph containing at least $m$ pairwise independent edges and the vertices of $G$ are randomly assigned to points in convex position, then, almost surely, every planar embedding of $G$ mapping vertices to their assigned points and edges to polygonal curves has at least $m / 20$ edges represented by curves with at least $m / 40^{3}$ bends.
\end{abstract}

\section{Introduction}

A planar embedding of a planar graph $G$ is a drawing of $G$ in the plane, where the edges are represented by simple continuous curves which intersect only at endpoints representing common vertices. By definition, every planar graph has a planar embedding. In 1948, Fáry showed that every planar graph has a straightline embedding, i.e., a planar embedding where every edge is represented by a single line segment. Numerous algorithms exist for constructing straight-line embeddings. These algorithms construct and report a set of locations for the vertices of $G$. The line segments representing the edges of $G$ are implicitly given by the locations of their endpoints.

In this paper, we consider the problem of constructing a planar embedding where vertices are mapped to prespecified fixed locations. If $\left\{v_{1}, \ldots, v_{n}\right\}$ is the vertex set of $G$ and $\left\{p_{1}, \ldots, p_{n}\right\}$ is the set of vertex locations, then each $v_{i}$ must be mapped to the point $p_{i}$. In most cases, the edges of $G$ can no longer be represented by disjoint line segments. However, they can be represented by noncrossing polygonal curves. What is the complexity of these polygonal curves, i.e., how many bends (internal vertices) must they have? By a slight modification of

\footnotetext{
* Supported by NSF grant CCR-94-24398, OTKA-T-020914, and by a PSC-CUNY Research Award.

** Supported by NSA grant MDA904-97-1-0018 and by DIMACS.
} 
the algorithm presented in Souvaine and Wenger [6], we obtain that if $G$ is a single path, then it has a planar embedding with a total of $O\left(n^{2}\right)$ bends. Here we prove a stronger result, which answers a question asked by Richard Pollack.

Theorem 1. Every planar graph on $n$ vertices admits a planar embedding which maps each vertex to an arbitrarily prespecified distinct location and each edge to a polygonal curve with $O(n)$ bends.

Moreover, such an embedding can be constructed in $O\left(n^{2}\right)$ time.

We apply an idea of Pach, Shahrokhi, and Szegedy [5] to show that the bound in Theorem 1 is tight. We say that two edges of a graph are independent, if they do not share an endpoint. A set of pairwise independent edges is often called a matching. A set of $n / 2$ pairwise independent edges in a graph with $n$ vertices is called a perfect matching.

Theorem 2. Let $G$ be a planar graph of $n$ vertices, $v_{1}, \ldots, v_{n}$, which contains at least $m$ pairwise independent edges and let $\left(p_{1}, \ldots, p_{n}\right)$ be a random permutation of the vertices of a convex n-gon.

Then, as $n$ tends to infinity, in every planar embedding of $G$ which maps $v_{i}$ to $p_{i}$ and the edges to polygonal curves, there are almost surely at least $m / 20$ edges represented by curves with at least $m / 40^{3}$ bends.

Note that a path of length $n$ or a perfect matching of $n$ vertices have $\lfloor n / 2\rfloor$ pairwise independent edges.

The nature of the problem drastically changes if we only require that $\left\{v_{1}, \ldots\right.$, $\left.v_{n}\right\}$ be mapped to a set $\left\{p_{1}, \ldots, p_{n}\right\}$ of points in general position but we do not insist on the particular order. It is known that in this case there always exists a straight-line embedding which maps $v_{i}$ to $p_{\pi(i)}, 1 \leq i \leq n$, for a suitable permutation $\pi$ (see [2], 4]).

\section{Embedding Algorithm - Proof of Theorem 1}

Let $G$ be a planar graph with vertex set $V=\left\{v_{1}, \ldots, v_{n}\right\}$, and let $\left\{p_{1}, \ldots, p_{n}\right\}$ be a set of $n$ points in the plane. In this section, we give an algorithm for constructing a planar embedding of $G$ such that vertex $v_{i}$ is represented by point $p_{i}$ and each edge of $G$ is represented by a polygonal curve with $O(n)$ bends (internal vertices.) Our algorithm runs in $O\left(n^{2}\right)$ time.

A Hamiltonian cycle is a cycle which visits each vertex of the graph exactly once. A planar embedding of a cycle divides the plane into a bounded and an unbounded component. If the edges are represented by polygonal curves, then the bounded component is a simple polygon.

The general outline of our algorithm is as follows. We first bound the number of bends in a polygonal curve which follows the boundary of a tree at constant distance under the $l_{1}$ metric (Lemma 1). We next assume that $G$ contains a Hamiltonian cycle and show how to construct a planar embedding of $G$ mapping each vertex $v_{i}$ to point $p_{i}$ (Lemma 2 ). Finally, we show how to add vertices and 
edges to a planar graph forming a new planar graph which has a Hamiltonian cycle (Lemma 3). Combining the last two results gives a proof of Theorem 1.

To construct the planar embedding of a graph containing a Hamiltonian cycle, we construct a polygonal, planar embedding of a tree whose leaves are the points $p_{1}, \ldots, p_{n}$. Curves representing the edges of the cycle will follow the boundary of this tree or one of its subtrees at a fixed distance. We use the $l_{1}$ metric to measure this distance. The distance between points $\left(x_{1}, y_{1}\right)$ and $\left(x_{2}, y_{2}\right)$ in the $l_{1}$ metric is $\left|x_{1}-x_{2}\right|+\left|y_{1}-y_{2}\right|$. Let $B_{\epsilon}$ be the ball of radius $\epsilon$ in the $l_{1}$ metric. The Minkowski sum of a point set $S$ and $B_{\epsilon}$, written $S+B_{\epsilon}$, is the set of all points which are at distance less than or equal to $\epsilon$ from some point in $S$.

Lemma 1. Let $T$ be a straight-line embedding of a tree with $N>1$ vertices. If $\epsilon>0$ is smaller than the $l_{1}$-distance between any line segment (edge) $e$ and any vertex of $T$ not incident to $e$, then $T+B_{\epsilon / 2}$ is a simple polygon whose boundary contains at most $4 N-2$ vertices.

Proof. Each vertex of the polygon $T+B_{\epsilon / 2}$ is either a vertex of $u+B_{\epsilon / 2}$ for some vertex $u$ of $T$ or lies on the intersection of the boundary of $e+B_{\epsilon / 2}$ and $e^{\prime}+B_{\epsilon / 2}$ for two adjacent edges $e, e^{\prime}$ of $T$. The adjacent edges $e$ and $e^{\prime}$ share some common endpoint $u$. Thus each vertex of the polygon $T+B_{\epsilon / 2}$ can be associated with a vertex $u$ of $T$. The number of vertices associated with $u$ is at most $2+d_{u}$ where $d_{u} \geq 1$ is the degree of $u$. A tree has $N-1$ edges so the sum of the degrees of all tree vertices is $2 N-2$. Thus summing $2+d_{u}$ over all vertices of the tree gives a bound of $4 N-2$ on the number of vertices of $T+B_{\epsilon / 2}$.

An embedding of a graph is outerplanar if it is a simple closed curve with some non-crossing internal diagonals. The unbounded face of such an embedding corresponds to the (uniquely determined) Hamiltonian cycle of the graph. A graph is outerplanar if it has an outerplanar embedding. Mapping the vertices of an outerplanar graph to vertices of a convex $n$-gon in the order (clockwise or counter-clockwise) that they appear in the Hamiltonian cycle and its edges to line segments between $n$-gon vertices gives a straight-line planar embedding of the outerplanar graph. Every planar graph containing a Hamiltonian cycle can be divided into two outerplanar graphs which have only the edges of the Hamiltonian cycle in common.

We are ready to construct the planar embedding of a graph containing a Hamiltonian cycle.

Lemma 2. Let $G$ be a planar graph of $n$ vertices, $v_{1}, \ldots, v_{n}$, containing a Hamiltonian cycle $C$ and let $\left\{p_{1}, \ldots, p_{n}\right\}$ be a set of $n$ distinct points in the plane. Graph $G$ has a planar embedding such that

(i) every vertex $v_{i}$ of $G$ is represented by the point $p_{i}$;

(ii) every edge of $G$ is represented by a polygonal curve with at most $8 n+9$ bends.

Moreover, such an embedding can be constructed in $O\left(n^{2}\right)$ time. 

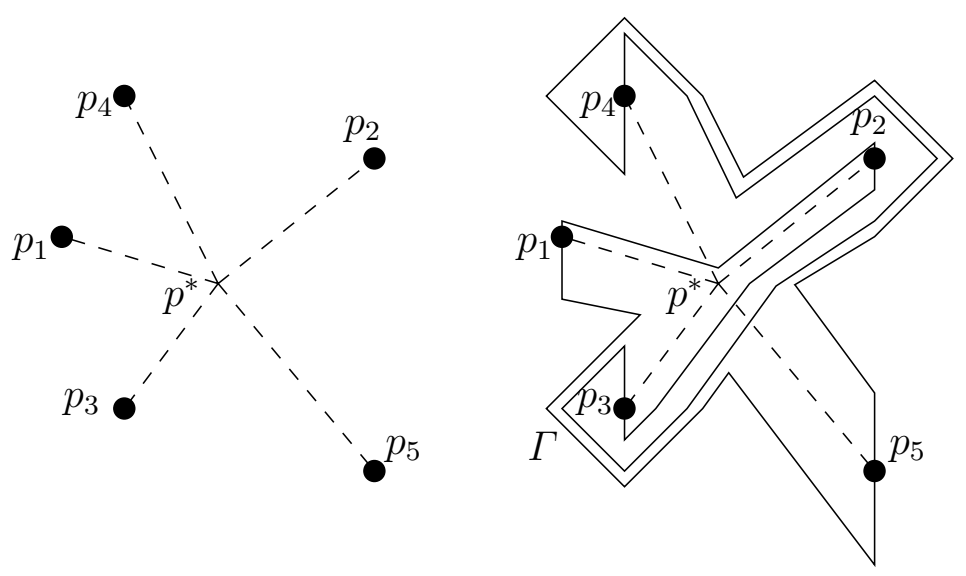

Fig. 1. Tree $T$ and the simple polygonal curve $\Gamma$ through $p_{1}, \ldots, p_{5}$.

Proof. By relabeling vertices and points, we may assume that the vertices of $G$ appear in the Hamiltonian cycle $C$ in the order $\left(v_{1}, \ldots, v_{n}\right)$. We first construct an embedding of the edges of $C$.

Let $p^{*}$ be some point in the plane to the right of $p_{1}$ and in general position with respect to all the points $p_{i}$, i.e., the line through any two distinct points, $p_{i}$ and $p_{j}$, does not contain $p^{*}$. In addition, $p^{*}$ should not share an $x$-coordinate with any of the points $p_{i}$. Let $T_{1}$ be the line segment $\left(p^{*}, p_{1}\right)$ and $T_{i}$ be the union of $T_{i-1}$ and $\left(p^{*}, p_{i}\right)$. Set $T$ equal to $T_{n}$. Note that the leaves of $T_{i}$ are $p_{1}, \ldots, p_{i}$ and that $T_{1} \subset T_{2} \subset \cdots \subset T_{n}=T$. The edges of the cycle will be routed around the embedded trees $T_{i}$.

Let $\epsilon>0$ be the smallest distance under the $l_{1}$ metric between any $p_{i}$ and any line segment $\left(p^{*}, p_{j}\right)$, where $j \neq i$. Let $\delta$ equal $\epsilon /(4 n)$. Draw a simple closed polygonal curve $\Gamma$ through the points $p_{1}, \ldots, p_{n}$ in sequential order as follows. Start by drawing a vertical line segment from $p_{1}$ to a point at distance $\delta$ above $p_{1}$. From this point, draw a polygonal curve clockwise around $T_{2}$ following the boundary of $T_{2}$ at a distance of $\delta$ in the $l_{1}$ metric. Stop when this polygonal curve crosses the vertical line through $p_{2}$ and draw a vertical line segment of length $3 \delta$ through $p_{2}$. The endpoint of the curve is now a distance $2 \delta$ from $p_{2}$.

For $i=2$ to $n-1$, draw a polygonal curve following the boundary of $T_{i+1}$ at distance of $i \delta$ in the $l_{1}$ metric. Stop when this the curve crosses the vertical line through $p_{i+1}$ and connect it to a vertical line segment of length $(2 i+1) \delta$ through $p_{i+1}$. There are two possible such curves, one clockwise and one counterclockwise around $T_{i+1}$. Choose the one which does not cover $p_{1}$. Note that at a distance of $i \delta$ from $T_{i+1}$, the curve will not intersect any of the previously drawn curves.

Complete $\Gamma$ by drawing a polygonal curve clockwise around $T$ at a distance of $n \delta$, and finally connecting the curve to $p_{1}$ by a vertical line segment below 
$p_{1}$. (See Figure[1, The simple closed polygonal curve $\Gamma$ will be the image of the Hamiltonian cycle $C$ in our embedding. Note that $p^{*}$ lies in the bounded region defined by $\Gamma$ and that the points $p_{1}, \ldots, p_{n}$ lie in clockwise order around $\Gamma$.

Except for the two line segments on either end, the curve from $p_{i}$ to $p_{i+1}$ is a subset of $T_{i}+B_{i \delta}$. Tree $T_{i}$ is a subtree of $T$ which has at most $n+1$ vertices. By Lemma 1, the curve from $p_{i}$ to $p_{i+1}$ has at most $(4(n+1)-2)+2=4(n+1)$ bends. Similarly, the curve from $p_{n}$ to $p_{1}$ has at most $4(n+1)$ bends.

We now construct pairwise disjoint auxiliary paths between the points $p_{i}$ and $p_{1}$. For positive integers $i \leq n$ and $k \leq n-1$, let $\Lambda_{i, k}$ be the boundary of $T_{i}+B_{(i-1) \delta+k \delta / n}$. The polygonal curve $\Lambda_{i, k}$ intersects $\Gamma$ at only two places, once on a line segment incident with $p_{i}$ and once on a line segment incident with $p_{1}$. (See Figure 2.) Thus, $\Gamma$ divides $\Lambda_{i, k}$ into two polygonal curves, one in the bounded region and one in the unbounded region defined by $\Gamma$. Truncate these curves at a distance $\delta$ from their endpoints and connect their new endpoints to $p_{i}$ and $p_{1}$ by line segments, thus forming two paths between $p_{i}$ and $p_{1}$. One of these paths lies in the interior of the bounded region and one in the interior of the unbounded region defined by $\Gamma$. Label these paths $\Lambda_{i, k}^{\prime}$ and $\Lambda_{i, k}^{\prime \prime}$ in the bounded and unbounded regions, respectively. These paths are pairwise disjoint except perhaps at their endpoints. Applying Lemma 1 shows that these paths have at most $4(n+1)$ bends.
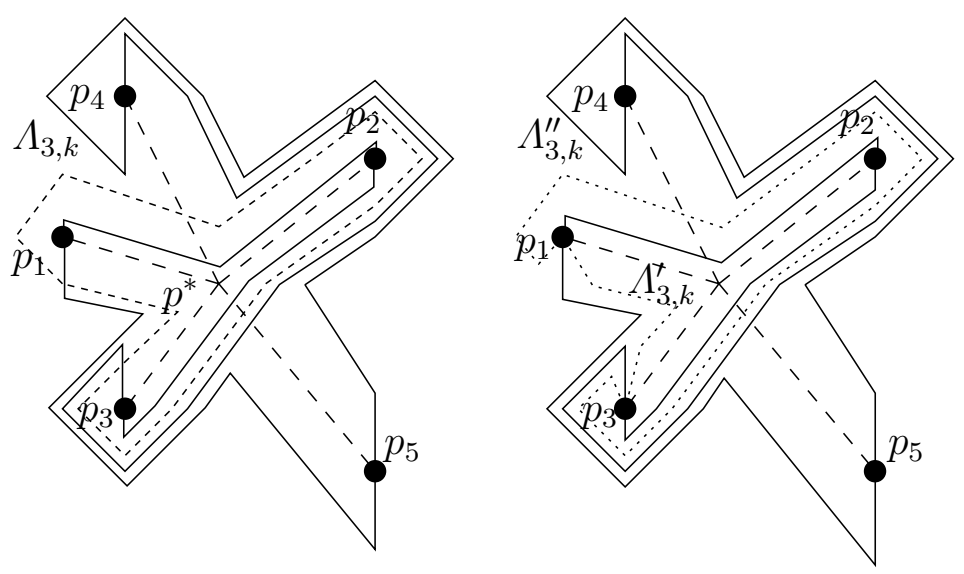

Fig. 2. The polygonal curve $\Lambda_{3, k}$ and paths $\Lambda_{3, k}^{\prime}$ and $\Lambda_{3, k}^{\prime \prime}$.

We are finally ready to embed the remaining edges of $G$. Divide $G$ into two outerplanar graphs $G^{\prime}$ and $G^{\prime \prime}$ which have only the edges of Hamiltonian cycle $C$ in common. Let $E^{\prime}$ and $E^{\prime \prime}$ be the edges of $G^{\prime}$ and $G^{\prime \prime}$, respectively, which are not in $C$.

Draw a circle $\Phi$ of radius $\delta / 2$ in the Euclidean norm around $p_{1}$ and intersect it with each of the $\Lambda_{i, k}^{\prime}$ and $\Lambda_{i, k}^{\prime \prime}$. Since the paths from $p_{1}$ to $p_{2}$ and $p_{n}$ start 
with vertical line segments above and below $p_{1}$, the curve $\Gamma$ divides $\Phi$ into two semi-circles, $\Phi^{\prime}$ and $\Phi^{\prime \prime}$ in the bounded and unbounded regions defined by $\Gamma$. Let $q_{i, k}^{\prime}$ and $q_{i, k}^{\prime \prime}$ be the intersection points of $\Lambda_{i, k}^{\prime}$ and $\Lambda_{i, k}^{\prime \prime}$ with $\Phi$, respectively.

For integers $i, j$, let $f(i, j)$ be the unique non-negative integer less than $n$ which is congruent to $(i-j) \bmod n$. We represent an edge $\left(v_{i}, v_{j}\right) \in E^{\prime}$ by a path from $p_{i}$ along $\Lambda_{i, f(i, j)}^{\prime}$ to $q_{i, f(i, j)}^{\prime}$, then a line segment from $q_{i, f(i, j)}^{\prime}$ to $q_{j, f(j, i)}^{\prime}$, and finally a path along $\Lambda_{j, f(j, i)}^{\prime}$ from $q_{j, f(j, i)}^{\prime}$ to $p_{j}$. This path contains at most $4(n+1)+4(n+1)+1=8 n+9$ bends. (See Figure 3 for an example of a complete embedding of a graph.)

We claim that this embedding of $G^{\prime}$ is planar. By construction, the paths $\Lambda_{i, k}^{\prime}$ intersect or touch $\Gamma$ only at their endpoints. Thus the only possible violations of planarity occur in the line segments that we drew in $\Phi^{\prime}$.

Since $G^{\prime}$ is outerplanar, mapping its vertices to vertices of a convex $n$-gon and its edges to a line segments between vertices gives a planar embedding of $G^{\prime}$. The points on $\Phi^{\prime}$ form vertices of a convex polygon and lie in the clockwise order

$$
p_{1}, q_{2,1}^{\prime}, \ldots, q_{2, n-1}^{\prime}, q_{3,1}^{\prime}, \ldots, q_{3, n-1}^{\prime}, \ldots, q_{n, 1}^{\prime}, \ldots, q_{n, n-1}^{\prime} .
$$

For each edge $\left(v_{i}, v_{j}\right)$ of $E^{\prime}$, we drew the line segment $\left(q_{i, f(i, j)}^{\prime}, q_{j, f(j, i)}^{\prime}\right)$ in $\Phi^{\prime}$. If instead we drew the line segment $\left(q_{i, 1}^{\prime}, q_{j, 1}^{\prime}\right)$ in the convex $n$-gon $\left(p_{1}, q_{1,1}^{\prime}, q_{2,1}^{\prime}, \ldots, q_{n, 1}^{\prime}\right)$ for each $\left(v_{i}, v_{j}\right) \in E^{\prime}$, we would have a planar embedding of $G^{\prime}$. If $\left(v_{i}, v_{j}\right)$ and $\left(v_{i^{\prime}}, v_{j^{\prime}}\right)$ are two edges in $E^{\prime}$ and $v_{i}, v_{j}, v_{i^{\prime}}$ and $v_{j^{\prime}}$ are distinct, then $q_{i, f(i, j)}^{\prime}, q_{j, f(j, i)}^{\prime}, q_{i^{\prime}, f\left(i^{\prime}, j^{\prime}\right)}^{\prime}$ and $q_{j^{\prime}, f\left(j^{\prime}, i^{\prime}\right)}^{\prime}$ lie in the same order around $\Phi^{\prime}$ as $q_{i, 1}^{\prime}, q_{j, 1}^{\prime}, q_{i^{\prime}, 1}^{\prime}, q_{j^{\prime}, 1}^{\prime}$. Since line segments $\left(q_{i, 1}^{\prime}, q_{j, 1}^{\prime}\right)$ and $\left(q_{i^{\prime}, 1}^{\prime}, q_{j^{\prime}, 1}^{\prime}\right)$ are pairwise disjoint, so are line segments $\left(q_{i, f(i, j)}^{\prime}, q_{j, f(j, i)}^{\prime}\right)$ and $\left(q_{i^{\prime}, f\left(i^{\prime}, j^{\prime}\right)}^{\prime}, q_{j^{\prime}, f\left(j^{\prime}, i^{\prime}\right)}^{\prime}\right)$. If $\left(v_{i}, v_{j}\right)$ and $\left(v_{i}, v_{j^{\prime}}\right)$ are two edges in $E^{\prime}$ sharing the vertex $v_{i}$, then the relative positions on $\Phi^{\prime}$ of $q_{i, f(i, j)}^{\prime}$ and $q_{i, f\left(i, j^{\prime}\right)}^{\prime}$ ensure that $\left(q_{i, f(i, j)}^{\prime}, q_{j, f(j, i)}^{\prime}\right)$ and $\left(q_{i, f\left(i, j^{\prime}\right)}^{\prime}, q_{j^{\prime}, f\left(j^{\prime}, i\right)}^{\prime}\right)$ are pairwise disjoint.

An edge $\left(v_{i}, v_{j}\right) \in E^{\prime \prime}$ is represented by a path from $p_{i}$ along $\Lambda_{i, f(i, j)}^{\prime \prime}$ to $q_{i, f(i, j)}^{\prime \prime}$, followed by a line segment from $q_{i, f(i, j)}^{\prime \prime}$ to $q_{j, f(j, i)}^{\prime \prime}$, and finally a path from $q_{j, f(j, i)}^{\prime \prime}$ to $p_{j}$. This path also contains at most $4(n+1)+4(n+1)+1=8 n+9$ line bends. A similar argument to the one for $E^{\prime}$ shows that this embedding of $E^{\prime \prime}$ is planar. The only difference is that the points lie in counterclockwise order around $\Phi^{\prime \prime}$.

Finding a point $p^{*}$ in general position and calculating the value of $\epsilon$ can be done in $O(n \log n)$ time. Constructing the polygonal curves from $p_{i-1}$ to $p_{i}$ and from $p_{n}$ to $p_{1}$ takes $O(n)$ time per curve. Constructing the polygonal curves representing edges in $E^{\prime}$ and $E^{\prime \prime}$ also take $O(n)$ time per edge for a total of $O\left(n^{2}\right)$ time.

We now show how to turn a planar graph $G$ into a planar graph $H$ containing a Hamiltonian cycle.

Lemma 3. Let $G$ be a planar graph of $n$ vertices. By subdividing edges of $G$ by at most two new vertices and adding some edges between vertices, we can 


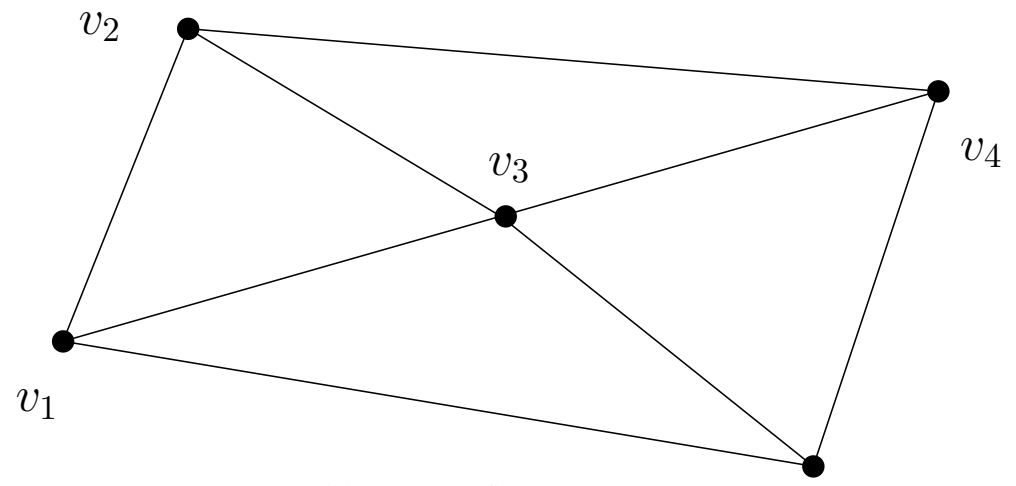

Graph $G$

$v_{5}$

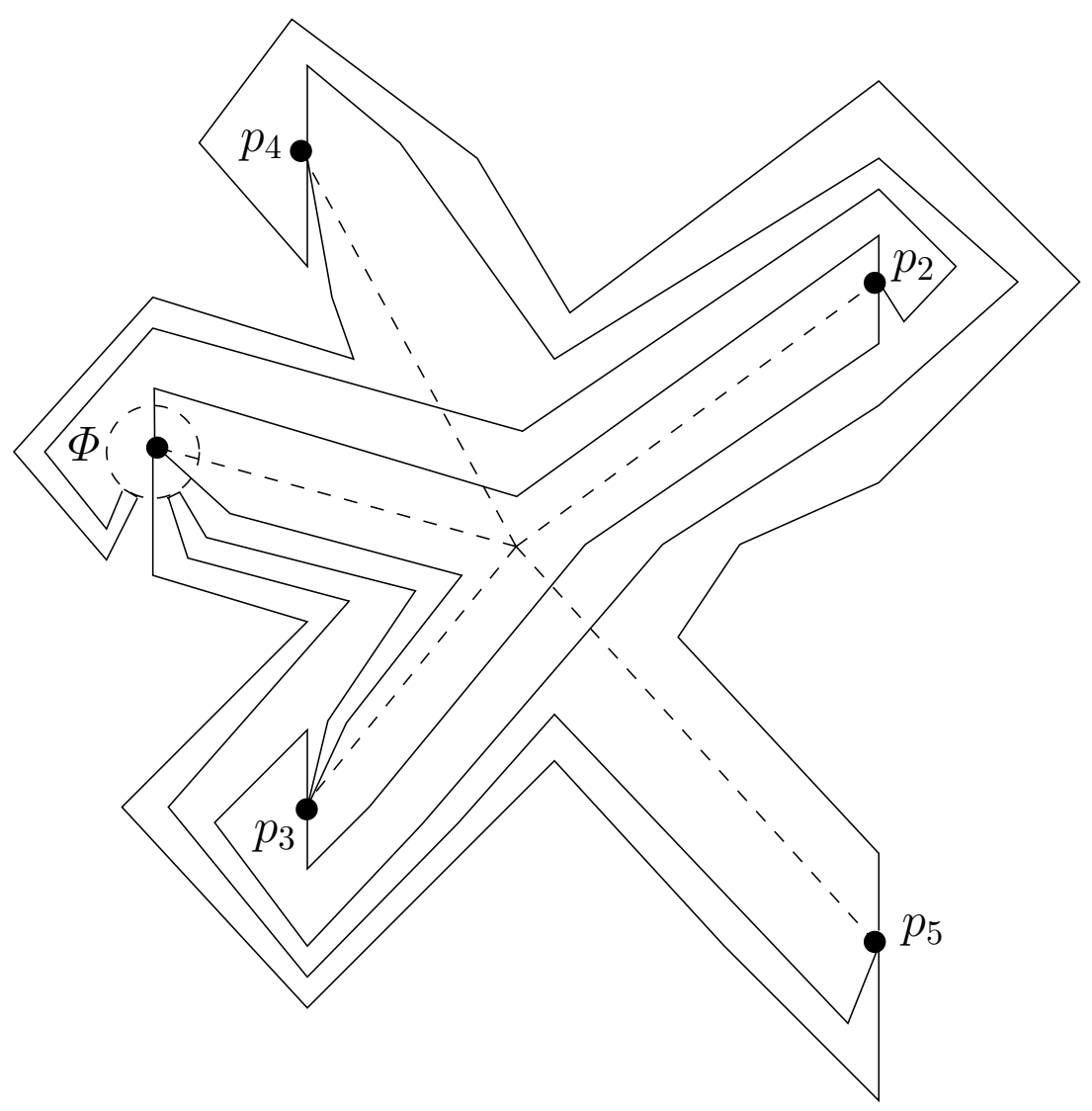

Fig. 3. Planar embedding of graph $G$ where vertex $v_{i}$ maps to point $p_{i}$. 
construct from $G$ a planar graph $H$ which has at most $5 n-10$ edges and contains a Hamiltonian cycle. This construction can be accomplished in linear time.

Proof. If $G$ has $k>1$ connected components, add $k-1$ new edges to form a connected graph $G^{\prime}$. Construct a planar embedding of $G^{\prime}$, not necessarily with straight-line edges. Let $S$ be a spanning tree of $G^{\prime}$. Clearly, all edges of $G^{\prime}-G$ belong to $S$. (See Figure 4 )
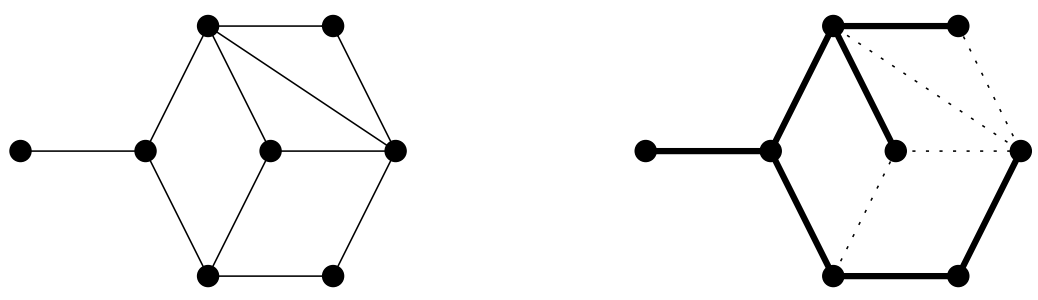

Fig. 4. Graph $G$ and a spanning tree $S$.

Starting at any vertex, walk clockwise around $S$, visiting its vertices in order. Note that the internal vertices of $S$ will be visited more than once. Label the vertices with $w_{1}, w_{2}, \ldots, w_{n}$, by the order, in which they are first visited. If $w_{i}$ and $w_{i+1}$ are connected by an edge, then let this edge belong to the Hamiltonian cycle $\left(1 \leq i \leq n\right.$, and we use the convention $\left.w_{n+1}:=w_{1}\right)$. If not, connect $w_{i}$ to $w_{i+1}$ by a simple curve clockwise around the boundary of $S$, passing very close to it. Wherever this curve intersects an edge of $G^{\prime}$, introduce a new vertex. Thus, this curve becomes a path whose pieces are added as edges to the graph and to its Hamiltonian cycle. Merge any multiple edges, and call the resulting graph $H$. (See Figure 5,
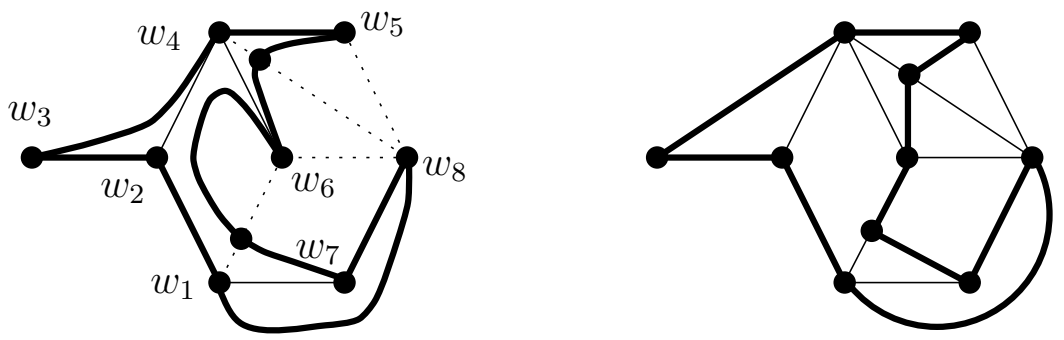

Fig. 5. Constructing the Hamiltonian cycle and graph $H$.

Each edge of $G^{\prime}$ was split at most twice. Since $G^{\prime}$ has at most $3 n-6$ edges and the $n-1$ edges of $S$ were never split, $H$ has at most $n+2(2 n-5)=5 n-10$ vertices. 
Connecting $G$ to form $G^{\prime}$, constructing a planar embedding and a spanning tree $S$ of $G^{\prime}$ take $O(n)$ time. Walking around $S$ to construct the Hamiltonian cycle can also be done in linear time.

Proof of Theorem 1: Starting with a planar graph $G$ of $n$ vertices, form a planar graph $H$ on at most $5 n-10$ vertices containing a Hamiltonian cycle as outlined in Lemma 3 Only the $n$ original vertices from $G$ have prespecified locations, but we can arbitrarily assign locations to the new vertices in $H$. Applying Lemma 2 gives a planar embedding of $H$, whose edges are polygonal curves with at most $8(5 n-10)+9<40 n$ bends. We construct an embedding of $G$ from the embedding of $H$ by merging polygonal curves which correspond to portions of edges of $G$ and by deleting polygonal curves which are not part of any edge of $G$. Each edge of $G$ was split into at most 3 edges in $H$, so each edge in $G$ can be represented by a polygonal curve with at most $3 * 40 n=120 n$ bends.

Constructing $H$ takes $O(n)$ time. Embedding $H$ in the plane takes $O\left(n^{2}\right)$ time. Constructing the embedding of $G$ from the embedding of $H$ takes time proportional to the size of the embedding of $H$ which is $O\left(n^{2}\right)$. Thus, our algorithm runs in $O\left(n^{2}\right)$ time.

A more generalized version of Lemma 2 is also true. Given a Hamiltonian, planar graph $H^{\prime}$ of $n$ vertices where only $k \geq 1$ vertices of $H^{\prime}$ have preassigned point locations, it is possible to represent every edge of $H^{\prime}$ by a polygonal curve with at most $8 k+9$ bends. Applying this bound to $H$ in the proof of Theorem 1 gives a planar embedding of $H$ with at most $8 n+9<8(n+2)$ bends per curve. This results in each edge of the original graph $G$ being represented by curves with at most $3 * 8(n+2)=24(n+2)$ bends.

\section{Lower Bound - Proof of Theorem 2}

Let $H$ be a graph with vertex set $V(H)$ and edge set $E(H)$. The bisection width $b(H)$ of $H$ is the minimum number of edges running between $V_{1}$ and $V_{2}$, over all partitions of the vertex set $V(H)$ into two parts $V_{1} \cup V_{2}$ such that $\left|V_{1}\right|,\left|V_{2}\right| \geq|V(H)| / 3$. Roughly speaking, the bisection width is the minimum number of edges whose deletion splits the graph into two approximately equal parts.

The crossing number $c(H)$ of $H$ is the minimum number of crossing pairs of arcs in any planar representation of $H$, where the vertices are mapped to distinct points and the edges are represented by simple continuous arcs connecting the corresponding points and not passing through the image of any other vertex.

Leighton [3] discovered that the above parameters are closely related. A somewhat more general form of his result was established in [5].

Lemma 4. Let $H$ be a graph of $n$ vertices, whose degrees are $d_{1}, \ldots, d_{n}$. Then

$$
b^{2}(G) \leq(1.58)^{2}\left(16 c(G)+\sum_{i=1}^{n} d_{i}^{2}\right) .
$$


The following lemma bounds the bisection width of a random graph which is constructed from the union of a cycle and a random perfect matching.

Lemma 5. Let $H_{n}$ be a randomly defined graph with vertex set $V\left(H_{n}\right)=$ $\left\{u_{1}, \ldots, u_{n}\right\}$, whose edge set is the union of $\left\{u_{1} u_{2}, u_{2} u_{3}, \ldots, u_{n} u_{1}\right\}$ and a random perfect matching of the vertices ( $n$ is even).

Then, as $n$ tends to infinity, the bisection width of $H_{n}$ is almost surely at least $n / 20$.

Proof. The number of different graphs that can be obtained as $H_{n}$ is equal to the number of perfect matchings on $n$ vertices, $\frac{n !}{(n / 2) ! 2^{n / 2}}$. All of these graphs are equally likely.

Next we estimate that at most how many of these graphs have bisection width at most $k$, for some fixed $k \leq n / 3$. Consider the cycle $C=u_{1} \ldots u_{n} \subseteq H_{n}$. The number of partitions $V\left(H_{n}\right)=V_{1} \cup V_{2}$, for which at most $k$ edges of $C$ run between $V_{1}$ and $V_{2}$, is at most

$$
\sum_{i=0}^{k}\left(\begin{array}{l}
n \\
i
\end{array}\right)<(k+1)\left(\begin{array}{l}
n \\
k
\end{array}\right)
$$

These edges cut $C$ into at most $k$ intervals, belonging alternately to $V_{1}$ and to $V_{2}$.

For a fixed partition with $2 n / 3 \geq\left|V_{1}\right|=n_{1} \geq\left|V_{2}\right|=n_{2} \geq n / 3$, the number of perfect matchings of $V(H)$ with at most $k$ edges running between $V_{1}$ and $V_{2}$ is at most

$$
\begin{gathered}
\sum_{i=0}^{k}\left(\begin{array}{c}
n_{1} \\
i
\end{array}\right)\left(\begin{array}{c}
n_{2} \\
i
\end{array}\right) i ! \frac{\left(n_{1}-i\right) !}{\left(\frac{n_{1}-i}{2}\right) ! 2^{\frac{n_{1}-i}{2}}} \frac{\left(n_{2}-i\right) !}{\left(\frac{n_{2}-i}{2}\right) ! 2^{\frac{n_{2}-i}{2}}} \\
>(k+1)\left(\begin{array}{c}
2 n / 3 \\
k
\end{array}\right)\left(\begin{array}{c}
n / 3 \\
k
\end{array}\right) k ! \frac{(2 n / 3-k) !}{\left(\frac{2 n / 3-k}{2}\right) ! 2^{\frac{2 n / 3-k}{2}}} \frac{(n / 3-k) !}{\left(\frac{n / 3-k}{2}\right) ! 2^{\frac{n / 3-k}{2}} .}
\end{gathered}
$$

Indeed, one can choose $i$ points from $V_{1}$ and $i$ points from $V_{2}$ in $\left(\begin{array}{c}n_{1} \\ i\end{array}\right)\left(\begin{array}{c}n_{2} \\ i\end{array}\right)$ different ways, and match them in $i$ ! ways. The number of matchings on the remaining $n_{j}-i$ points of $V_{j}$ is at most $\frac{\left(n_{j}-i\right) !}{\left(\frac{n_{j}-i}{2}\right) ! 2 \frac{n_{j}-i}{2}} ; j=1,2$. Thus, the probability that $b\left(H_{n}\right) \leq k$ is at most

$$
(k+1)^{2}\left(\begin{array}{c}
n \\
k
\end{array}\right)\left(\begin{array}{c}
2 n / 3 \\
k
\end{array}\right)\left(\begin{array}{c}
n / 3 \\
k
\end{array}\right) k ! \frac{(2 n / 3-k) !}{\left(\frac{2 n / 3-k}{2}\right) ! 2^{\frac{2 n / 3-k}{2}}} \frac{(n / 3-k) !}{\left(\frac{n / 3-k}{2}\right) ! 2^{\frac{n / 3-k}{2}}} / \frac{n !}{(n / 2) ! 2^{n / 2}},
$$

which tends to zero when $k=\lfloor n / 20\rfloor$ and $n \rightarrow \infty$.

Proof of Theorem 2 : Let $P=\left\{p_{1}, \ldots, p_{n}\right\}$ be the set of vertices of a convex $n$ gon in the plane, listed in clockwise order. Let $G$ be a planar graph on the vertex set $V(G)=\left\{v_{1}, \ldots, v_{n}\right\}$, and let $f: V(G) \rightarrow P$ be a randomly chosen bijection. Suppose that $G$ has $m$ pairwise independent edges, $v_{1} v_{2}, \ldots, v_{2 m-1} v_{2 m}$. 
Let $H_{2 m}$ be a graph on the vertex set $V\left(H_{2 m}\right)=\left\{f\left(v_{1}\right), \ldots, f\left(v_{2 m}\right)\right\}$, constructed as follows. If $f\left(v_{i_{1}}\right), \ldots, f\left(v_{i_{2 m}}\right)$ is the list the elements of $V\left(H_{2 m}\right)$ in clockwise order around $P$, then let

$$
E\left(H_{2 m}\right)=\left\{f\left(v_{i_{j}}\right) f\left(v_{i_{j+1}}\right) \mid 1 \leq j \leq 2 m\right\} \cup\left\{f\left(v_{2 i-1}\right) f\left(v_{2 i}\right) \mid 1 \leq i \leq m\right\},
$$

where $i_{2 m+1}:=i_{1}$. Clearly, $H_{2 m}$ is isomorphic to the graph described in Lemma 5. In particular, almost surely we have $b\left(H_{2 m}\right) \geq m / 10$.

Suppose now, in order to obtain a contradiction, that $G$ has a planar embedding which maps $v_{i}$ to $f\left(v_{i}\right), 1 \leq i \leq n$, and every edge is represented by a polygonal curve such that at most $m / 20$ edges are represented by curves with at least $m / 40^{3}$ bends. If, for some $1 \leq i \leq m, v_{2 i-1} v_{2 i}$ is represented by a curve with at least $m / 40^{3}$ bends, then remove $f\left(v_{2 i-1}\right) f\left(v_{2 i}\right)$ from the graph $H_{2 m}$. The bisection width of the resulting graph $H_{2 m}^{\prime}$ almost surely satisfies

$$
b\left(H_{2 m}^{\prime}\right) \geq b\left(H_{2 m}\right)-m / 20 \geq m / 10-m / 20=m / 20 .
$$

Hence, applying Lemma 4 with $d_{i} \leq 3(1 \leq i \leq m)$, we obtain that almost surely

$$
c\left(H_{m}^{\prime}\right) \geq \frac{1}{16}\left(\frac{b^{2}\left(H_{m}^{\prime}\right)}{(1.58)^{2}}-9 m\right) \geq \frac{(m / 20)^{2}}{40}-m=\frac{m^{2}}{40 \times 20^{2}}-m .
$$

On the other hand, in the above planar embedding of $G$, all paths representing the edges of $H_{m}^{\prime}$ have fewer than $m / 40^{3}$ bends. Adding the edges of the convex hull of $f\left(v_{i_{1}}\right), \ldots, f\left(v_{i_{2 m}}\right)$ to the collection of these paths, we obtain a planar representation of $H_{2 m}^{\prime}$ with at most $m \times\left(m / 40^{3}+1\right) \times 2 \leq 2 m^{2} / 40^{3}+2 m$ crossings, because each line segment in a polygonal path can cross at most two edges of the convex hull of $P$. Thus,

$$
\frac{m^{2}}{40 \times 20^{2}}-m \leq c\left(H_{m}^{\prime}\right) \leq \frac{m^{2}}{40^{2} \times 20}+2 m,
$$

a contradiction for suitably large $m$.

\section{Remarks}

This paper discusses the worst case complexity of constructing a polygonal planar embedding of a graph $G$ of $n$ vertices, $v_{1}, \ldots, v_{n}$, where each vertex $v_{i}$ is mapped to a prespecified point $p_{i}$. The corresponding optimization problem is the following: given a planar graph $G$ with vertex set $\left\{v_{1}, \ldots, v_{n}\right\}$ and a point set $\left\{p_{1}, \ldots, p_{n}\right\}$, construct an embedding of $G$ which maps $v_{i}$ to $p_{i}, 1 \leq i \leq n$, using as few bends (in total) as possible. Bastert and Fekete 1] proved that this problem is NP-hard. Is there an approximation algorithm for this problem which gives a solution within a factor of the optimal one?

Bastert and Fekete actually proved that minimizing the total number of bends is NP-hard in the case when $G$ is a perfect matching. However, the complexity of the problem is unknown if $G$ is a simple path, or, more generally, 
any connected graph. The complexity is also unknown if the points $p_{1}, \ldots, p_{n}$ are required to be in convex position (vertices of a convex polygon.) Are these restrictions of the problem also NP-hard?

The $\Omega\left(n^{2}\right)$ worst case lower bound in Theorem 2 assumes that the points $p_{1}, \ldots, p_{n}$ are in convex position. What if the points are not in convex position, say, they form a $\sqrt{n} \times \sqrt{n}$ grid? Are there examples of assignments of vertices of a planar graph $G$ to the vertices of such a grid such that any polygonal planar embedding of $G$ requires a total of $\Omega\left(n^{2}\right)$ bends?

\section{References}

1. Bastert, O., And Fekete, S. Geometrische Verdrahtungsprobleme. Technical Report 96.247, Angewandte Mathematik und Informatik, Universität zu Köln, Köln, Germany, 1996.

2. Gritzmann, P., Mohar, B., Pach, J., and Pollack, R. Embedding a planar triangulation with vertices at specified points. American Mathematical Monthly 98 (1991), 165-166. (Solution to problem E3341).

3. Leighton, F. Complexity Issues in VLSI. MIT Press, Cambridge, 1983.

4. Pach, J., And Agarwal, P. Combinatorial Geometry. John Wiley and Sons, New York, 1995.

5. Pach, J., Shahrokhi, F., And Szegedy, M. Applications of the crossing number. Algorithmica 16 (1996), 111-117. (Special Issue on Graph Drawing, edited by G. Di Battista and R. Tamassia).

6. Souvaine, D., And Wenger, R. Constructing piecewise linear homeomorphisms. Technical Report 94-52, DIMACS, New Brunswick, New Jersey, 1994. 